\title{
(2) OPEN ACCESS \\ Sharing a household with children and risk of COVID-19: a study of over 300000 adults living in healthcare worker households in Scotland
}

\author{
Rachael Wood ㅇ , 1,2 Emma Thomson, ${ }^{3}$ Robert Galbraith, ${ }^{4}$ Ciara Gribben, \\ David Caldwell, ${ }^{5}$ Jennifer Bishop, ${ }^{5}$ Martin Reid, ${ }^{1}$ Anoop S V Shah, ${ }^{6}$ Kate Templeton, ${ }^{7}$ \\ David Goldberg, ${ }^{5}$ Chris Robertson, ${ }^{5}$ Sharon J Hutchinson, ${ }^{5}$ Helen M Colhoun (1) , ${ }^{5,8}$ \\ Paul M McKeigue (10, ${ }^{2}$ David A McAllister (1) ${ }^{5,9}$
}

\section{- Additional material is published online only. To view, please visit the journal online (http://dx.doi.org/10.1136/ archdischild-2021-321604) \\ 'Public Health Scotland, Edinburgh, UK \\ ${ }^{2}$ Usher Institute, University of Edinburgh, Edinburgh, UK ${ }^{3} \mathrm{MRC}$ Centre for Virus Research, University of Glasgow, Glasgow, UK \\ ${ }^{4}$ Retired, Edinburgh, UK ${ }^{5}$ Public health Scotland Glasgow, UK \\ ${ }^{6}$ Department of Non- communicable Disease Epidemiology, London School of Hygiene \& Tropical Medicine, London, UK \\ ${ }^{7}$ Department of Medical Microbiology, Royal Infirmary of Edinburgh, Edinburgh, UK ${ }^{8}$ Institute of Genetics and Molecular Medicine, The University of Edinburgh, Edinburgh, UK \\ ${ }^{9}$ Institute of Health and Wellbeing, University of Glasgow, Glasgow, UK}

\section{Correspondence to} Dr David A McAllister, David McAllister, University of Glasgow School of Medicine, Glasgow G12 8QQ, UK: david.mcallister@glasgow.ac.uk

Received 11 January 2021 Revised 16 February 2021 Accepted 17 February 2021 18 March 2021 Published Online First

\section{ABSTRACT}

Objective Children are relatively protected from COVID-19, due to a range of potential mechanisms. We investigated if contact with children also affords adults a degree of protection from COVID-19.

Design Cohort study based on linked administrative data.

Setting Scotland.

Study population All National Health Service Scotland healthcare workers and their household contacts as of March 2020.

Main exposure Number of young children (0-11 years) living in the participant's household.

Main outcomes COVID-19 requiring hospitalisation, and any COVID-19 (any positive test for SARS-CoV-2) in adults aged $\geq 18$ years between 1 March and 12 October 2020.

Results 241 266, 41 198, 23783 and 3850 adults shared a household with 0, 1, 2 and 3 or more young children, respectively. Over the study period, the risk of COVID-19 requiring hospitalisation was reduced progressively with increasing numbers of household children - fully adjusted HR (aHR) 0.93 per child ( $95 \% \mathrm{Cl} 0.79$ to 1.10$)$. The risk of any COVID-19 was similarly reduced, with the association being statistically significant (aHR per child 0.93; 95\% Cl 0.88 to 0.98). After schools reopened to all children in August 2020, no association was seen between exposure to young children and risk of any COVID-19 (aHR per child 1.03; 95\% Cl 0.92 to 1.14).

Conclusion Between March and October 2020, living with young children was associated with an attenuated risk of any COVID-19 and COVID-19 requiring hospitalisation among adults living in healthcare worker households. There was no evidence that living with young children increased adults' risk of COVID-19, including during the period after schools reopened.

\section{Check for updates}

(c) Author(s) (or their employer(s)) 2021. Re-use permitted under CC BY. Published by BMJ.

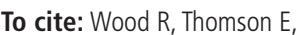
Galbraith $\mathrm{R}$, et al.

Arch Dis Child

2021:106:1212-1217.

\section{INTRODUCTION}

To date, children seem to be relatively protected from SARS-CoV-2, being substantially less likely to develop symptomatic infection (COVID-19) or serious illness. ${ }^{12}$ Zimmermann and $\mathrm{Curtis}^{3}$ recently provided a wide-ranging review of the proposed mechanisms for this difference, including factors such as concurrent infections competing with SARS-CoV-2 in vivo, differences in microbiota,

\section{What is already known on this topic?}

- Young children are less likely to develop COVID-19 and severe COVID-19 than adults, due to a range of potential mechanisms, among which is the possibility of cross-protective immunity to SARS-CoV-2.

- Whether contact with young children offers adults a degree of protection from COVID-19 or not is unknown.

- Two studies have suggested that contact with children may be inversely associated with COVID-19 risk (risk of infection, hospitalisation or death from COVID-19).

\section{What this study adds?}

- In our large cohort, adults with young children were at lower risk of testing positive for SARS-CoV-2 and possibly also of developing COVID-19 requiring hospitalisation.

- Adults living with young children were not at increased risk of COVID-19, including during August-October 2020 when nurseries and schools were open for all children.

- Verification of this finding is warranted in other populations and settings.

higher melatonin levels and lower intensity of exposure to SARS-CoV-2. Differences in innate and/or acquired immune mechanisms may play a part, ${ }^{3-11}$ as might increased innate immune responsiveness following vaccinations and high exposure to respiratory viruses. ${ }^{3}{ }^{12}$ Alternatively, pre-exposure to antigenically similar infectious agents (providing subsequent specific cross-protection against SARSCoV-2) may also be relevant. Children have higher levels of exposure to endemic coronaviruses than adults. ${ }^{13} 14$ Evidence exists for $\mathrm{B}$ cell and $\mathrm{T}$ cell cross-reactivity between SARS-CoV-2 and endemic coronaviruses, ${ }^{4-10}$ and SARS-CoV-2 responsive T cells have been shown to provide protection against COVID-19. ${ }^{11}$

Given this latter possibility, we reasoned that adults who are close contacts of children might also be protected from SARS-CoV-2 due to a degree of 
Table 1 Characteristics of adult household contacts by number of young children

\begin{tabular}{|c|c|c|c|c|}
\hline & 0 children aged $0-11$ years & 1 child aged $0-11$ years & 2 children aged $0-11$ years & $3+$ children aged $0-11$ years \\
\hline Number of adults & 241266 & 41198 & 23783 & 3850 \\
\hline Adults who are healthcare workers & $121004(50.15)$ & $22025(53.46)$ & $13179(55.41)$ & $2237(58.10)$ \\
\hline Age, mean (SD) & $44.53(15.04)$ & $39.82(10.58)$ & $39.01(8.08)$ & $38.47(6.62)$ \\
\hline Male & $105116(43.57)$ & $17639(42.82)$ & $10803(45.42)$ & $1783(46.31)$ \\
\hline \multicolumn{5}{|l|}{ Scottish index of multiple deprivation } \\
\hline 1 - most deprived & $37242(15.44)$ & $5655(13.73)$ & $2447(10.29)$ & $373(9.69)$ \\
\hline 2 & $46147(19.13)$ & $7700(18.69)$ & $3599(15.13)$ & $582(15.12)$ \\
\hline 3 & $48659(20.17)$ & $7491(18.18)$ & $4280(18.00)$ & 706 (18.34) \\
\hline 4 & $52847(21.90)$ & $9701(23.55)$ & $6102(25.66)$ & $999(25.95)$ \\
\hline 5 - least deprived & $56371(23.36)$ & $10651(25.85)$ & $7355(30.93)$ & $1190(30.91)$ \\
\hline Race/ethnicity - non-white & $7768(3.22)$ & $2088(5.07)$ & $1162(4.89)$ & $259(6.73)$ \\
\hline \multicolumn{5}{|l|}{ Comorbidity count } \\
\hline None & $207796(86.13)$ & $37315(90.57)$ & $21924(92.18)$ & $3564(92.57)$ \\
\hline One & $24897(10.32)$ & $3231(7.84)$ & $1579(6.64)$ & $252(6.55)$ \\
\hline Two or more & $8573(3.55)$ & $652(1.58)$ & $280(1.18)$ & $34(0.88)$ \\
\hline \multicolumn{5}{|c|}{ Occupation of healthcare worker in household } \\
\hline Nursing and midwifery & $102514(42.49)$ & $18688(45.36)$ & $10085(42.40)$ & $1530(39.74)$ \\
\hline Administrative services & 44929 (18.62) & $6710(16.29)$ & $3236(13.61)$ & $404(10.49)$ \\
\hline Support services & $27294(11.31)$ & $3232(7.85)$ & $1386(5.83)$ & $236(6.13)$ \\
\hline Medical and dental & $20836(8.64)$ & $4326(10.50)$ & $3586(15.08)$ & $849(22.05)$ \\
\hline Allied health profession & $20007(8.29)$ & $3798(9.22)$ & $2974(12.50)$ & $442(11.48)$ \\
\hline Other & $25686(10.65)$ & $4444(10.79)$ & $2516(10.58)$ & $389(10.10)$ \\
\hline \multicolumn{5}{|c|}{ Occupational role of healthcare worker in household } \\
\hline Non-patient facing & $50441(20.91)$ & $7453(18.09)$ & $3667(15.42)$ & $471(12.23)$ \\
\hline Patient facing & $137697(57.07)$ & $25461(61.80)$ & $15485(65.11)$ & $2627(68.23)$ \\
\hline Undetermined & $53128(22.02)$ & $8284(20.11)$ & $4631(19.47)$ & $752(19.53)$ \\
\hline \multicolumn{5}{|c|}{ Part time working in healthcare worker in household } \\
\hline Whole time & $147608(61.18)$ & $19482(47.29)$ & $8296(34.88)$ & $1206(31.32)$ \\
\hline Part time & 88351 (36.62) & $20329(49.34)$ & $14183(59.64)$ & $2281(59.25)$ \\
\hline Not recorded & $5307(2.20)$ & $1387(3.37)$ & $1304(5.48)$ & $363(9.43)$ \\
\hline Tested for SARS-CoV-2 & $14736(6.11)$ & $2835(6.88)$ & $1823(7.67)$ & 354 (9.19) \\
\hline
\end{tabular}

Statistics are the number (percentage) of adults with each characteristic except for age, which is given as the mean and SD.

immune cross-protection. This could be relevant to decisions to close nurseries and schools in response to SARS-CoV-2 ${ }^{15}$ and teachers' perceptions of workplace safety. ${ }^{16}$ Since few relevant studies exist, ${ }^{17}$ we used a recently reported cohort of around 160000 healthcare workers and 250000 household members in Scotland ${ }^{18}$ to test the hypothesis that risk of COVID-19 in adults is attenuated among those living with young children.

\section{METHODS}

\section{Population, data sources and record linkage}

Full details of the population studied are reported elsewhere. ${ }^{18}$ In brief, we identified healthcare workers in Scotland (aged 18-65 years) using databases including all individuals directly employed by the National Health Service (NHS) and all general practitioners providing services to the NHS, as of March 2020. We identified other members of the healthcare workers' households (all ages) using the NHS Scotland master patient index and exact address matching. We linked these data to multiple Scotland-wide databases indicating virology testing for SARSCoV-2, hospitalisation, critical care admission and death. Exposure, covariate and outcome data were examined for all adults aged $\geq 18$ years living in a healthcare worker household. All data from 1 March to 12 October 2020 were included.

\section{Outcome}

The primary outcome was COVID-19 requiring hospitalisation, defined as a first positive PCR test ${ }^{19}$ for SARS-CoV-2 up to 28 days prior to, or during, a hospital admission. Secondary outcomes were any COVID-19 (defined as any positive test for SARS-CoV-2) and severe COVID-19 (defined as a positive test for SARS-CoV-2 up to 28 days prior to admission for critical care or death).

\section{Exposure}

The primary exposure was the number of young children (aged 0-11 years) in each household. Additional analyses examined risk by the number of preschool children (aged 0-4 years), primary school children (aged 5-11 years), older children (aged $12-17$ years) and other adults (aged $\geq 18$ years).

\section{Covariates}

Data on age, sex and deprivation (Scottish Index of Multiple Deprivation quintile) were obtained from the linked databases. Prespecified comorbidities (see online supplemental table S1) were defined using previous hospitalisation and prescribing data. Ethnicity was imputed from forename and surname using the ONOMAP algorithm. ${ }^{20}$

Occupational covariates were defined at the household level based on the characteristics of the member who was a healthcare worker. These included the healthcare worker's occupation (eg, medical and nursing), potential exposure to SARS-CoV-2 (eg, patient-facing role or not), seniority, length of service, immigration status and full/part-time working status. In households with more than one healthcare worker, the highest risk category was 

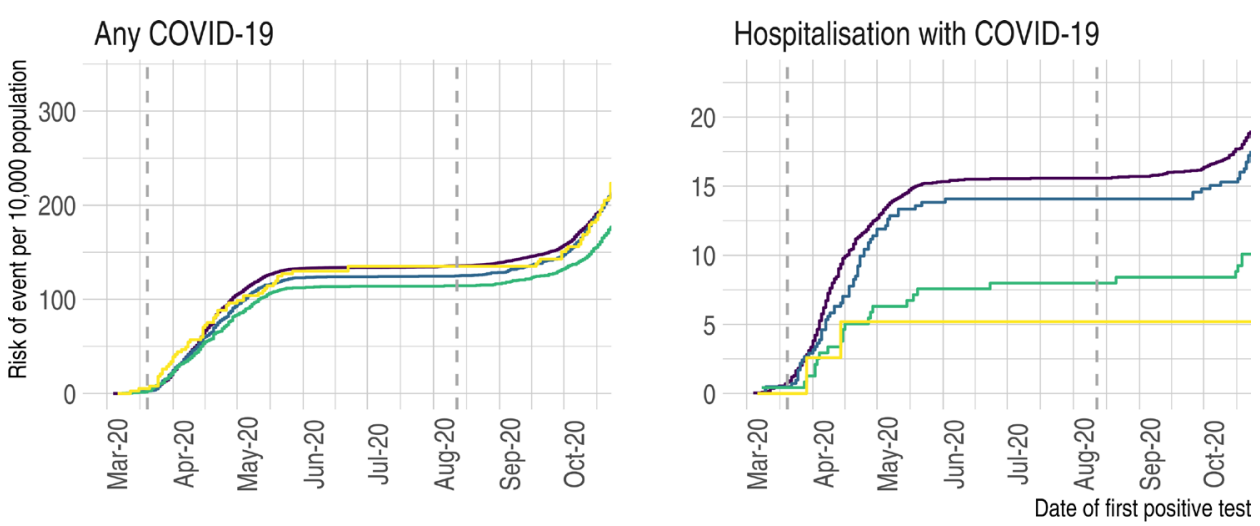

Number of children under 12 in household $-0-1-2-3$ or above

Figure 1 Risk of any COVID-19 and of COVID-19 requiring hospitalisation in adults living in healthcare worker households by number of young children (aged 0-11 years) cumulative incidence (risk) plots of any COVID-19 and of COVID-19 requiring hospitalisation by number of young children (aged 0-11 years) in household. Vertical dotted lines indicate dates when schools closed and reopened.

applied. A causal diagram showing the assumed relation between these covariates is provided in online supplemental figure S1.

\section{Statistical analysis}

We plotted the cumulative incidence of hospitalisation for COVID-19 among adults according to the number of young children in each household. We modelled COVID-19 requiring hospitalisation, any COVID-19 and severe COVID-19 using Cox regression, calculating robust SEs to allow for clustering due to shared household membership and stratifying on groups of health board areas to allow for differences in baseline hazard. We present effect estimates for minimal models adjusting for age, full models including all covariates and intermediate models. We conducted a range of sensitivity analyses incorporating additional covariates and/or restricting the population examined.

\section{RESULTS}

Of the 310097 adults living in a healthcare worker household, 241266 (78\%), 41198 (13\%), 23783 (7.8\%) and 3850 (1.2\%) shared their household with $0,1,2$ and 3 or more young children, respectively. Compared with adults living with no young children, those living with children were on average 5 years younger; were less likely to live in the most deprived areas; were more likely to be of non-white ethnicity; and were less likely to have comorbidities (table 1 and online supplemental table S1). Adults living with young children were slightly more likely to be tested for SARS-CoV-2 (table 1).
Household composition differed according to the number of young children. Households with more children were more likely to include two or more adults. More than a quarter of adults who shared a household with a single child under 11 ears also shared a household with a child aged $12-17$ years (online supplemental table S2).

\section{COVID-19 requiring hospitalisation}

Compared with adults living with no young children, the risk of COVID-19 requiring hospitalisation was reduced in those living with children (figure 1). The unadjusted HR for COVID-19 requiring hospitalisation was 0.77 per each additional young child in the household (95\% CI 0.65 to 0.90 , table 2). On adjusting for adults' age, this association was attenuated (HR per child $0.88 ; 95 \%$ CI 0.75 to 1.04 ). Further smaller changes were seen after adjusting for other potential confounders (sex, deprivation, occupation, professional role, staff/non-staff status, length of service, number of adolescents and adults in household, comorbidity count plus selected comorbidities (see online supplemental table S1) and full-time/part-time working status), with the fully adjusted HR (aHR) being 0.93 per child $(95 \% \mathrm{CI}$ 0.79 to 1.10 ).

Similar associations were found when analysis was restricted to households where at least one adult was a patient-facing healthcare worker (aHR per child $0.89 ; 95 \%$ CI 0.74 to 1.08 , online supplemental table S3). In a stratified analysis, the aHR per child was 1.11 (95\% CI 0.92 to 1.35 ) for adults living in

Table 2 Risk and HRs for COVID-19 requiring hospitalisation for adults living in healthcare worker households by number of young children

\begin{tabular}{|c|c|c|c|c|c|}
\hline & No children aged $0-11$ years & 1 child aged $0-11$ years & 2 children aged $0-11$ years & $3+$ children aged $0-11$ years & Per child \\
\hline $\begin{array}{l}N \text { adults with COVID-19 requiring } \\
\text { hospitalisation }\end{array}$ & 390 & 59 & 20 & 2 & - \\
\hline Total $\mathrm{N}$ adults & 241266 & 41198 & 23783 & 3850 & - \\
\hline Risk per 10000 & 16.2 & 14.3 & 8.4 & 5.2 & - \\
\hline Unadjusted & 1 & $0.89(0.67-1.17)$ & $0.53(0.34-0.83)$ & $0.33(0.08-1.32)$ & $0.77(0.65-0.90)$ \\
\hline Model 1 & 1 & $1.09(0.82-1.46)$ & $0.69(0.44-1.10)$ & $0.44(0.11-1.79)$ & $0.88(0.75-1.04)$ \\
\hline Model 2 & 1 & $1.11(0.83-1.49)$ & $0.72(0.45-1.15)$ & $0.44(0.11-1.78)$ & $0.90(0.76-1.05)$ \\
\hline Model 3 & 1 & $1.13(0.84-1.52)$ & $0.73(0.46-1.16)$ & $0.45(0.11-1.81)$ & $0.90(0.77-1.06)$ \\
\hline Model 4 & 1 & $1.17(0.87-1.57)$ & $0.78(0.49-1.26)$ & $0.49(0.12-1.97)$ & $0.93(0.79-1.10)$ \\
\hline
\end{tabular}

HRs obtained from Cox proportional hazard models. Model 1 adjusts for adults' age using a penalised spline function. Model 2 additionally adjusts for sex, Scottish Index of Multiple Deprivation quintile, occupation (eg, nursing and medical), occupational role (patient facing, non-patient facing and undetermined), healthcare worker (yes/no), length of service, number of children aged 12-17 years in household and number of adults in household. Model 3 additionally adjusts for the comorbidity count and specific conditions (ischaemic heart disease, other heart disease, other circulatory system diseases, advanced chronic kidney disease, asthma and chronic lower respiratory disease, neurological disorders, decompensated liver disease, any immunological condition, malignant neoplasms, disorders of oesophagus, stomach and duodenum, type 1 diabetes and type 2 diabetes). Model 4 additionally adjusts for part-time status (additional model not prespecified). 
Table 3 Risk and HRs for any COVID-19 for adults living in healthcare worker households by number of young children

\begin{tabular}{|c|c|c|c|c|c|}
\hline & $\begin{array}{l}\text { No children aged 0-11 } \\
\text { years }\end{array}$ & 1 child aged $0-11$ years & 2 children aged $0-11$ years & $\begin{array}{l}3+\text { children aged 0-11 } \\
\text { years }\end{array}$ & Per child \\
\hline $\mathrm{N}$ adults with any COVID-19 & 4324 & 717 & 345 & 65 & - \\
\hline Total $\mathrm{N}$ adults & 241266 & 41198 & 23783 & 3850 & - \\
\hline Risk per 10000 & 179.2 & 174.0 & 145.1 & 168.8 & - \\
\hline Unadjusted & 1 & $0.97(0.89-1.06)$ & $0.81(0.72-0.92)$ & $0.96(0.73-1.26)$ & $0.93(0.89-0.98)$ \\
\hline Model 1 & 1 & $0.93(0.85-1.02)$ & $0.78(0.69-0.88)$ & $0.91(0.69-1.21)$ & $0.91(0.87-0.96)$ \\
\hline Model 2 & 1 & $0.93(0.85-1.02)$ & $0.79(0.70-0.90)$ & $0.90(0.68-1.18)$ & $0.92(0.87-0.96)$ \\
\hline Model 3 & 1 & $0.93(0.85-1.02)$ & $0.79(0.70-0.90)$ & $0.90(0.68-1.18)$ & $0.92(0.87-0.96)$ \\
\hline Model 4 & 1 & $0.94(0.86-1.03)$ & $0.82(0.72-0.93)$ & $0.92(0.70-1.22)$ & $0.93(0.88-0.98)$ \\
\hline
\end{tabular}

Model fitting and covariates as per footnote of table 2.

households containing a full-time healthcare worker and 0.65 (95\% CI 0.46 to 0.91 ) for those in households containing a parttime healthcare worker $(\mathrm{p}$ interaction $=0.002)$. A further stratified analysis found similar results for adults with (aHR 0.91 ; $95 \%$ CI 0.75 to 1.10 ) and without (aHR $0.90 ; 95 \%$ CI 0.65 to $1.25)$ any comorbidities.

\section{Any COVID-19}

Compared with adults living with no young children, the risk of any COVID-19 was reduced in those living with children (figure 1). In the full study population, the fully aHR for any COVID-19 was similar to that seen for COVID-19 requiring hospitalisation but with narrower CIs (aHR per child 0.93 95\% CI 0.88 to 0.98 , table 3 ). The inverse association between number of young children in the household and risk of any COVID-19 was similar in adults with (aHR per child $0.84 ; 95 \%$ CI 0.72 to 0.99 ) and without (aHR 0.93 ; $95 \%$ CI 0.88 to 0.97 ) comorbidities and in adults living in households containing a full-time (aHR 0.95 ; 95\% CI 0.88 to 1.02 ) and part-time (aHR 0.88; $95 \%$ CI 0.81 to 0.94 ) healthcare worker ( $\mathrm{p}$ interaction $=0.44$ ).

Further analysis of the risk of any COVID-19 revealed stronger inverse associations for adults living with preschool children, compared with those living with primary school children, adolescents or other adults (table 4). Similar differences between the age groups, but with wider $95 \%$ CIs reflecting the smaller numbers of events, were also found for the primary outcome of COVID-19 requiring hospitalisation (online supplemental table S4).

\section{School reopening}

Nurseries and schools in Scotland were closed from 23 March 2020 until the end of the school year in late June 2020. They reopened to all children on 12 August 2020 and remained open until the Christmas holiday. Some childcare or school provision was available for children of key workers, including healthcare workers, between March and August. Between 12 August and 12 October 2020 (the end date of our study period), there were an additional 1337 cases of any COVID-19 and 20 cases of COVID-19 requiring hospitalisation among the adults in our study population (figure 1), and the aHR for any COVID-19 was 1.03 (95\% CI 0.92 to 1.14 ) per each additional young child in the household.

\section{Additional analyses}

Results for the much less common outcome, severe COVID-19, are shown in online supplemental tables S5 and S6). Restricting the analysis to individuals identified via the ONOMAP algorithm as white did not modify the associations for any of the outcomes. The full set of regression coefficients and SEs for all fitted models are provided at https://github.com/ChronicDiseaseEpi/hcw.

\section{DISCUSSION}

We examined the hypothesis that the risk of COVID-19 in adults is attenuated for those with high exposure to young children (0-11 years) due to presumed cross-protective immunity. Among a cohort of over 300000 adults living in a household with a healthcare worker in Scotland, the risk of testing positive for SARS-CoV-2 over the period March-October 2020 was slightly lower for individuals living with young children, and this reduction persisted after adjusting for potential confounders. The risk of COVID-19 requiring hospitalisation (primary outcome specified) was also lower for those living with young children, although this finding did not reach statistical significance.

Very few studies have directly examined whether contact with children affords adults protection from SARS-CoV-2. Prior to our recent preprint, ${ }^{21}$ only one study touching on this question was identified. ${ }^{17}$ In this German study, 1186 of 4010 patients who had recovered from COVID-19 responded to a survey. The proportion of individuals reporting regular contact with children aged $<11$ years was lower than expected based on general population rates. More recently a preprint has been published by the OpenSAFELY group. ${ }^{22}$ In this general population sample of 12 million people in England, between February and July 2020,

Table 4 HRs for any COVID-19 for adults living in healthcare worker households by number of persons of different ages

\begin{tabular}{lllll}
\hline & Per child aged 0-4 years & Per child aged 5-11 years & Per child aged 12-17 years & Per adult aged 18 years or above \\
\hline Unadjusted & $0.88(0.81-0.96)$ & $1.07(1.01-1.13)$ & $0.87(0.84-0.89)$ & $0.97(0.91-1.02)$ \\
Model 1 & $0.87(0.80-0.95)$ & $1.03(0.97-1.09)$ & $0.88(0.85-0.90)$ & $0.94(0.88-0.99)$ \\
Model 2 & $0.86(0.78-0.94)$ & $1.05(0.99-1.12)$ & $1.04(1.02-1.07)$ & $0.95(0.89-1.01)$ \\
Model 3 & $0.86(0.78-0.94)$ & $1.05(0.99-1.12)$ & $1.04(1.02-1.07)$ & $0.95(0.89-1.01)$ \\
Model 4 & $0.87(0.80-0.95)$ & $1.06(1.00-1.12)$ & $1.04(1.01-1.07)$ & $0.96(0.90-1.02)$ \\
\hline
\end{tabular}

Model specification and covariates as per footnote of table 2. The unadjusted models and model 1 were fitted separately for each exposure (eg, aged 0-5 years and aged 6-11 years), but all exposures were included in models 2, 3 and 4 . The effect estimates corresponds to 'per child' column in tables $2-3$, where the counts of children and adults were treated as continuous variables, which assumes that any association between the number of children (or adults) and the hazard rate is log-linear. 
adults sharing a household with children aged 0-11 years were not at increased risk of any COVID-19 or COVID-19 requiring hospitalisation or critical care admission and were at a lower risk of death from COVID-19. These studies are congruent with our findings and together suggest that, to date, exposure to young children is not associated with increased risk of COVID-19 in adults but with a small reduction in risk.

The risk to adults of COVID-19 will presumably reflect a dynamic balance between the risk that children may directly transmit SARS-CoV-2 to their adult contacts, and the possibility that they may enhance cross-protective immunity through prior transmission of other respiratory viruses. In this context, our current study provides reassurance that adults sharing a household with young children remained at no increased risk of COVID-19 during August-October 2020 when schools were reopened and community transmission of SARS-CoV-2 was occurring.

This study has some limitations. The observed inverse association between living with young children and adults' risk of COVID-19 was not strong and could be a chance finding. Our primary outcome, COVID-19 requiring hospitalisation, was uncommon; hence, HR CIs were wide. Although we suspected that statistical power would be limited, we prespecified this as the primary outcome as we were concerned that high rates of (non-SARS-CoV-2) acute respiratory infection in households with small children might have led to higher levels of testing for SARS-CoV-2 and hence biased ascertainment of any COVID19. The level of testing was indeed higher among those adults who shared a household with young children. However, point estimates for COVID-19 requiring hospitalisation and any COVID-19 were similar, and for the latter, they were statistically significant.

Another possibility is that, despite extensive adjustment for potential confounders, the observed inverse association may be the result of residual confounding. On stratified analyses, the inverse association was evident for adults living in part-time healthcare worker households but less obvious for those living in full-time healthcare worker households. Since part-time workers with increasing numbers of children likely work fewer hours (and therefore have lower occupational exposure to SARS-CoV-2), and since we lacked accurate data on hours worked during the pandemic, we cannot exclude the possibility of unmeasured confounding as a cause of the observed associations.

It has been suggested that the inverse association between living with young children and risk of COVID-19 may result from adults living with children spending less time outside the home in settings in which SARS-CoV-2 may be transmitted. While plausible, we are unable to find any empirical evidence in support of this view. For much of the pandemic across much of Scotland, many social venues have been closed. In addition, evidence from the 2018 Scottish Household Survey (online supplemental tables S7-10) suggests that adults who live with young children are as likely to visit restaurants and gyms as other adults of the same age and indeed more likely to visit places of worship and cinemas.

If a protective effect of children on COVID-19 rate and severity in their adult contacts is confirmed, this could involve cross-reactive immunity to endemic COVID-19 infections acquired outside the home, for example, at nursery or school. First, evidence of antigenic similarity between $\mathrm{N}$ proteins of SARS-CoV-2 and those of endemic beta coronaviruses (strains Cov-OC43 and Cov-NL63) has now been shown in studies of cell-mediated immunity. There is also evidence of cross-reactivity in antibody-mediated immunity, although it is uncertain how well this protects against COVID-19. ${ }^{4-10}$ Second, respiratory samples obtained from children during investigation for respiratory tract infections show high levels of seasonal, endemic coronaviruses. ${ }^{13} 14$ Third, as well as having higher rates of exposure to such viruses, children may transmit seasonal coronaviruses to their household contacts. Younger adults (aged 15-44 years) that include those most likely to live with young children have higher levels of antibodies to $\mathrm{N}$ proteins of CoV-OC43 than do older adults, ${ }^{23}$ although whether this reflects exposure at home via contact with children, or elsewhere, is unknown. It would be important to compare the prevalence of antibodies to SARS$\mathrm{CoV}-2$, and to seasonal coronaviruses, in those with and without substantive exposure to children of different age groups.

Moreover, it is important to note that differences in previous exposure to other coronaviruses is only one of a range of potential explanations for the difference in COVID-19 risk among adults and children, for most of which-such as differences in melatonin levels-there are no plausible mechanisms by which adult contacts could share in the protection. ${ }^{3}$

Notwithstanding possible mechanisms, our findings provide sufficient evidence of a potentially interesting protective effect against COVID-19 for adults living with young children to warrant further study in other populations (eg, adults working in nurseries and primary schools) and settings. Ongoing work to explore whether potential protective effects persist as community transmission patterns evolve, in particular in response to the emergence of new viral strains and the implementation of vaccination programmes, would also be beneficial.

\section{CONCLUSION}

In a large occupational cohort, household exposure to young children was associated with a reduced risk of testing positive for SARS-CoV-2 and COVID-19 requiring hospitalisation (non-significant). Verification of this finding is needed in other populations and settings. Less equivocally, to date, increased household exposure to young children has not been associated with an increased risk of COVID-19, even during periods where schools are open and there is active transmission of SARS-CoV2 in the community. These findings have potential for informing policy on nursery and school closure.

Contributors DM, RW, ET, RG, KT, CR, SJH, HC and PM made substantial contributions to the conception or design of the work. DM, CG, DC, JB, MR and PM made substantial contributions to the analysis of data for the work. DM, RW, ET, RG, ASVS, DG, CR, SJH, HC, PM and DM made substantial contributions to the interpretation of data for the work. DM, RW and RG drafted the work. All authors revised it critically for important intellectual content, gave approval of the version to be published and gave their agreement to be accountable for all aspects of the work in ensuring that questions related to the accuracy or integrity of any part of the work are appropriately investigated and resolved.

Funding David McAllister is funded via a Wellcome Trust Intermediate Clinical Fellowship and Beit Fellowship (201492/Z/16/Z). Anoop Shah is funded via the British Heart Foundation through an Intermediate Clinical Research Fellowship (FS/19/17/34172).

Competing interests AS is co-owner of two nurseries.

Patient consent for publication Not required.

Ethics approval This project was approved by the Public Benefit and Privacy Panel (2021-0013).

Provenance and peer review Not commissioned; externally peer reviewed

Data availability statement Data may be obtained from a third party and are not publicly available. Data may be accessed via a secure platform following successful application to the Public Benefit and Privacy Panel via application to the electronic Data Research Information Services of Public Health Scotland.

Supplemental material This content has been supplied by the author(s). It has not been vetted by BMJ Publishing Group Limited (BMJ) and may not have 
been peer-reviewed. Any opinions or recommendations discussed are solely those of the author(s) and are not endorsed by BMJ. BMJ disclaims all liability and responsibility arising from any reliance placed on the content. Where the content includes any translated material, BMJ does not warrant the accuracy and reliability of the translations (including but not limited to local regulations, clinical guidelines, terminology, drug names and drug dosages), and is not responsible for any error and/or omissions arising from translation and adaptation or otherwise.

Open access This is an open access article distributed in accordance with the Creative Commons Attribution 4.0 Unported (CC BY 4.0) license, which permits others to copy, redistribute, remix, transform and build upon this work for any purpose, provided the original work is properly cited, a link to the licence is given, and indication of whether changes were made. See: https://creativecommons.org/ licenses/by/4.01.

\section{ORCID iDs}

Rachael Wood http://orcid.org/0000-0003-4453-623X

Helen M Colhoun http://orcid.org/0000-0002-8345-3288

Paul M McKeigue http://orcid.org/0000-0002-5217-1034

David A McAllister http://orcid.org/0000-0003-3550-1764

\section{REFERENCES}

1 Swann OV, Holden KA, Turtle L, et al. Clinical characteristics of children and young people admitted to hospital with covid-19 in United Kingdom: prospective multicentre observational cohort study. BMJ 2020;370:m3249.

2 Spiegelhalter D. What have been the fatal risks of Covid, particularly to children and younger adults? [Internet]. Medium, 2020. Available: https://medium.com/ wintoncentre/what-have-been-the-fatal-risks-of-covid-particularly-to-children-andyounger-adults-a5cbf7060c49

3 Zimmermann P, Curtis N. Why is COVID-19 less severe in children? A review of the proposed mechanisms underlying the age-related difference in severity of SARSCoV-2 infections. Arch Dis Child 2021;106:429-39.

4 Le Bert N, Tan AT, Kunasegaran K, et al. SARS-CoV-2-specific T cell immunity in cases of COVID-19 and SARS, and uninfected controls. Nature 2020;584:1-10.

5 Grifoni A, Weiskopf D, Ramirez SI, et al. Targets of T cell responses to SARS-CoV-2 coronavirus in humans with COVID-19 disease and unexposed individuals. Cell 2020;181:1489-501.

6 Lv H, Wu NC, Tsang OT-Y, et al. Cross-reactive antibody response between SARSCoV-2 and SARS-CoV infections. Cell Rep 2020:31:107725.

7 van der Heide V, Heide vander V. SARS-CoV-2 cross-reactivity in healthy donors. Nat Rev Immunol 2020;20:408.

8 KW N, Faulkner N, Cornish GH. Pre-existing and de novo humoral immunity to SARSCoV-2 in humans. bioRxiv 2020. doi:10.1101/2020.05.14.095414
9 Pia L. SARS-CoV-2-reactive T cells in patients and healthy donors. Nat Rev Immunol 2020;20:353.

10 Huang AT, Garcia-Carreras B, Hitchings MDT, et al. A systematic review of antibody mediated immunity to coronaviruses: antibody kinetics, correlates of protection, and association of antibody responses with severity of disease. medRxiv 2020. doi:10.110 1/2020.04.14.20065771. [Epub ahead of print: 17 Apr 2020].

11 Wyllie D, Mulchandani R, Jones HE. SARS-CoV-2 responsive T cell numbers are associated with protection from COVID-19: a prospective cohort study in keyworkers. medRxiv 2020

12 Blok BA, Arts RJW, van Crevel $R$, van $C R$, et al. Trained innate immunity as underlying mechanism for the long-term, nonspecific effects of vaccines. J Leukoc Biol 2015;98:347-56.

13 Gaunt ER, Hardie A, Claas ECJ, et al. Epidemiology and clinical presentations of the four human coronaviruses 229E, HKU1, NL63, and OC43 detected over 3 years using a novel multiplex real-time PCR method. I Clin Microbiol 2010;48:2940-7.

14 Nickbakhsh S, Ho A, Marques DFP, et al. Epidemiology of seasonal coronaviruses: establishing the context for the emergence of coronavirus disease 2019. J Infect Dis 2020;222:17-25.

15 Viner RM, Russell SJ, Croker H, et al. School closure and management practices during coronavirus outbreaks including COVID-19: a rapid systematic review. Lancet Child Adolesc Health 2020;4:397-404

16 EIS. Reopening schools survey results [Internet]. Available: https://www.eis.org.uk/ Coronavirus/ReopeningSchoolsSurvey [Accessed 17 Aug 2020].

17 Dugas M, Schrempf I-M, Ochs K, et al. Association of contact to small children with a mild course of COVID-19. Int I Infect Dis 2020;100:314-5.

18 Shah ASV, Wood R, Gribben C, et al. Risk of hospital admission with coronavirus disease 2019 in healthcare workers and their households: nationwide linkage cohort study. BMJ 2020;371:m3582.

19 Williams TC, Wastnedge E, MCAllister G, et al. Sensitivity of RT-PCR testing of upper respiratory tract samples for SARS-CoV-2 in hospitalised patients: a retrospective cohort study. Wellcome Open Res 2020;5:254.

20 Lakha F, Gorman DR, Mateos P. Name analysis to classify populations by ethnicity in public health: validation of Onomap in Scotland. Public Health 2011:125:688-96

21 Wood R, Thomson EC, Galbraith R. Sharing a household with children and risk of COVID-19: a study of over 300000 adults living in healthcare worker households in Scotland. medRxiv 2020.

22 Forbes $\mathrm{H}$, Morton CE, Bacon S. Association between living with children and outcomes from COVID-19: an OpenSAFELY cohort study of 12 million adults in England. medRxiv 2020

23 Gao X, Zhou H, Wu C, et al. Antibody against nucleocapsid protein predicts susceptibility to human coronavirus infection. J Infect 2015;71:599-602. 